\title{
EL ESTILO DIDÁCTICO BASADO EN EL CONTENIDO Y SU IMPLICANCIA EN EL DESARROLLO DE LA CAPACIDAD DE INVESTIGACIÓN EN LA FACULTAD DE CIENCIAS EMPRESARIALES.UPT 2014
}

\section{The teaching style based on the content and its implication in the development of research capacity at the Faculty of Business. UPT 2014}

Luis Celerino Catacora Lira ${ }^{6}$

\section{RESUMEN}

Objetivo: La presente investigación tuvo como objetivo determinar el tipo de implicancias que genera los estilos didácticos con énfasis en el contenido en el nivel de desarrollo de la capacidad de investigación en estudiantes de la Escuela Profesional de Ingeniería Comercial de la Facultad de Ciencias Empresariales 2014.

Método: La investigación es descriptiva explicativa y parte de la premisa de que los estilos didácticos de los profesores universitarios tienen influencia en el desarrollo de la capacidad de investigación de los estudiantes. La población estuvo integrada por los estudiantes de la escuela profesional de Ingeniería Comercial y la muestra por los grupos de estudiantes del cuarto y octavo ciclo de la carrera universitaria.

Resultados: Este documento presenta los resultados del predominio del estilo didáctico tradicional en el desarrollo de la capacidad de investigación de los estudiantes universitarios. La investigación se realizó en la Escuela Profesional de Ingeniería Comercial componente de la Facultad de Ciencias Empresariales de la Universidad Privada de Tacna. El estudio permitió comprobar que el estilo predominante del profesor universitario fue el tradicional, que se caracteriza por dar bastante énfasis al contenido, por explicar durante toda la clase y por que el profesor es el único que habla en la clase. Por el lado de los estudiantes, se llegó a comprobar que los estudiantes no han logrado desarrollar su capacidad de investigación.

\footnotetext{
${ }^{6}$ Doctor en ciencias de la educación
}

Conclusión: Los resultados de la investigación permitió comprobar con el $95 \%$ de confianza que el estilo didáctico tradicional que aplicaron los profesores universitarios durante el ciclo académico 2014 tuvo influencia significa sobre el nivel de desarrollo de la capacidad de investigación de los estudiantes, cuyo efecto se tradujo en la limitada elaboración y divulgación de notas científicas desde las aulas.

Palabras claves: Estilos didácticos, capacidad de investigación, divulgación científica

\section{ABSTRAC}

Objective: This study aimed to determine the type of implications that generates teaching styles with emphasis on the content development level research capacity in students from the Professional School of Business, Engineering 2014. Method: The research is descriptive and explanatory part of the premise that teaching styles of university professors have influence on the development of research capacity of students. The population consisted of students of vocational school Commercial Engineering and sample groups of students in the fourth and eighth cycle of the university.

Results: This paper presents the results of the dominance of traditional teaching style in developing research capacity of university students. The research was conducted at the Professional School of Business Administration component of the Faculty of Business Studies of the Private University of Tacna. The study allowed to verify that the predominant style was the traditional university professor, characterized by giving enough emphasis to content, to explain the entire class and the teacher is the only one talking in class. On 
the side of students, he came to see that students have failed to develop their research capacity.

Conclusion: The results of the research allowed check with $95 \%$ confidence that the traditional teaching style applied university professors during the academic year 2014 had influence means on the level of development of the research capacity of students, whose effect It resulted in limited development and dissemination of scientific notes from the classroom.

Keywords: Teaching Styles, research ability, scientific disclosure

\section{INTRODUCCIÓN}

En las universidades del mundo se forman estudiantes para resolver los grandes problemas de la sociedad así como también para investigar y buscar nuevas explicaciones a los problemas que afectan el desarrollo del país. Los profesores universitarios conocedores de esta misión se convierten en los principales actores del proceso de formación de los estudiantes. Pero también es muy importante saber que cada profesor tiene su propio estilo de enseñar. Muchos de ellos no son pedagogos pero aún así ellos actúan con algún estilo didáctico que frecuentemente los caracteriza. La presente investigación partió de la pregunta de si los estilos didácticos de los profesores tienen alguna influencia en el desarrollo de la capacidad de investigación de los estudiantes. Para ellos fue vital trabajar con los estudiantes, quienes son los protagonistas del proceso de aprendizaje en las aulas. En ese sentido se eligió una muestra con dos grupos de ellos, uno del cuarto ciclo que están casi por la mitad de la carrera profesional y los de octavo ciclo que son los que están a puertas de egresar.

La experiencia nos permitió conocer que el estilo didáctico que predominó en los profesores fue el estilo tradicional, con un fuerte énfasis en la enseñanza de los contenidos, en la que el profesor es la máxima autoridad y en la que las ideas de los estudiantes no son relevantes. Este descubrimiento del estilo predominante, nos indujo a verificar si tiene alguna influencia en el desarrollo de la capacidad de investigación de los estudiantes. Utilizando un cuestionario con la escala de Lickert para cada una de las variables, se obtuvo datos que nos permitió confirmar que los estilos didácticos de los profesores si tienen influencia sobre la capacidad de investigación de los estudiantes.

Para confirmar el nivel de influencia que presentaban ambas variables se vio por conveniente aplicar la prueba estadística del Chi cuadrado. Los resultados del análisis estadístico permitieron verificar con un nivel de confianza del 95\% que el estilo didáctico tradicional si afecto el desarrollo de la capacidad de investigación de los estudiantes. Deduciendo como consecuencia que los estudiantes en su formación profesional no reciben de sus profesores el aprendizaje de cómo escribir ensayos, monografías y notas científicas.

El modelo de enseñanza, también denominado tradicional, se caracteriza por el énfasis en la transmisión de información, asumiendo el profesor el lugar protagónico, tratando de inculcar nociones e introducirlas en la memoria del alumno, concebido éste como receptáculo y depositario del conocimiento. Es una educación vertical y autoritaria o paternalista que predomina en el sistema educativo universitario.

Sustenta su influencia educativa en el modelo de comunicación mono lógico o transmisor, basado en la existencia clásica de un emisor y un receptor. La información transita esencialmente del profesor (emisor) al alumno (receptor), caracterizándose por ser unidireccional, por lo que no se establece en este caso un verdadero proceso de comunicación, que implica la alternancia de estas funciones. La participación del estudiante en 
este modelo de comunicación, se refiere generalmente a la reproducción de las palabras del maestro o del texto.

En este modelo la comunicación se concibe como un instrumento valioso para la educación, pero no constituye la esencia de la misma, otorgándosele especial importancia a las técnicas comunicativas utilizadas por el profesor como emisor; así, todos los recursos que tienen que ver con el dominio del discurso oral, que permiten contribuir a la instrucción y educación tal y como son concebidas en este modelo, tributan a los objetivos propuestos.

La Pedagogía Tradicional constituye la tendencia pedagógica representativa de este primer modelo de educación. Es muy frecuente su aplicación en docentes que no tienen formación pedagógica. Al respecto Carmen Páez, sostiene: "La mayoría de los modelos tradicionales se centraban en el profesorado y en los contenidos. Los aspectos metodológicos, el contexto y, especialmente, el alumnado, quedaban en un segundo plano. El modelo didáctico tradicional pretende formar a los alumnos dándoles a conocer las informaciones fundamentales de la cultura vigente. Los contenidos se conciben desde una perspectiva más bien enciclopédica y con un carácter acumulativo. El conocimiento sería una especie de selección divulgativa de lo producido por la investigación científica, plasmado en los manuales universitarios “.

Los ejes centrales de esta concepción lo constituyen el texto y el profesor. Se enfatiza el contenido, en el texto y en la transmisión de conocimientos y valores a través de la palabra del profesor. Se pretende la memorización de un gran volumen de información. Se le exige al profesor una gran preparación académica, con un gran dominio de su materia; en eso estriba fundamentalmente su autoridad ante los estudiantes y la sociedad. También se le exigen determinadas cualidades personales que le permitan hacer uso de su autoridad y a la vez mantener el orden y la disciplina deseados. Al alumno le corresponde obedecer, escuchar y ser depositario del conocimiento. Su actividad se limita en cierta medida a la memorización sin una debida reflexión crítica.

En el devenir de su desarrollo hasta la actualidad, la tendencia pedagógica tradicional ha incorporado diversos elementos de otras tendencias: del empirismo, conductismo, humanismo, por lo que en la práctica pedagógica contemporánea no se manifiesta en su forma clásica y mantiene gran influencia, no sólo en nuestro país, sino también en otras latitudes.

\section{El modelo didáctico tradicional}

\section{A. ¿Qué se enseña?}

$>\quad$ Se enseñan los contenidos que se conciben, desde una perspectiva enciclopédica y con un carácter acumulativo y tendiente a la fragmentación, siendo la referencia única la disciplina; es decir, el conocimiento sería una especie de selección divulgativa de lo producido por la investigación científica, plasmado en los textos.

$>\quad$ Se enseña los contenidos esenciales de una determinada asignatura. Estos contenidos son provenientes de la cultura vigente de forma fragmentada, con un carácter acumulativo.

$>\quad$ Los conocimientos disciplinares es un conjunto de datos, conceptos y teorías, que han superado la prueba de veracidad científica, que se trasmiten de manera separada de las experiencias de los estudiantes y de las realidades sociales.

$>\quad$ El conocimiento disciplinar es jerárquicamente superior a cualquier otra forma de conocimiento, se utiliza para educar, y va acompañado de la realización de una serie de actividades o de de ejercicios con una intención de refuerzo o de ilustración de lo expuesto de manera eminentemente conceptual. 


\section{B. Ideas e intereses de los estudiantes}

No se tienen en cuenta ni los intereses ni las ideas de los estudiantes.

Las ideas y opiniones de los alumnos, si es que existen, serán sustituidas mediante este proceso por las ideas correctas del profesor.

Los estudiantes son actores pasivos de la clase, se encargan sólo de tomar nota por escrito de toda la información que suministra el profesor ya sea oral o escrito.

En ciertos casos este proceso se completa con algunos momentos esporádicos dedicados a dialogar sobre el tema, a realizar problemas de aplicación o a poner en marcha actividades prácticas de comprobación.

$>\quad$ Las concepciones de los estudiantes, los puntos de vista no científicos, o la información proveniente del trabajo directo de la realidad, no son contenidos relevantes a tener en cuenta en las clases.

$>\quad$ Los estudiantes se caracterizan por su poca iniciativa, inseguridad, escaso interés personal.

\section{C. ¿Cómo enseñar?}

El profesor es un ejecutor de directivas preestablecidas.

El profesor es autoritario, rígido y controlador.

El alumno es un sujeto pasivo, reproductor del conocimiento.

El método de enseñanza se limita, a una exposición, lo más ordenada y clara posible, de "lo que hay que enseñar", con el apoyo de un libro de texto como recurso único o, al menos, básico; ello puede ir acompañado de la realización de una serie de actividades, con una intención de refuerzo o de ilustración de lo expuesto, y en todo caso ateniéndose a la lógica, eminentemente conceptual, del conocimiento que se intenta transmitir.

Para enseñar el docente organiza sus actividades en torno a una secuencia de temas que pretende ser una selección pormenorizada de lo que el estudiante debe saber sobre la disciplina.

$>\quad$ El profesor consume una parte importante del tiempo explicando los temas. El método de enseñanza se limita a una exposición, lo más ordenada y clara posible, de lo que hay que enseñar, con el apoyo del libro de texto como recurso y acompañada de actividades y ejercicios de reforzamiento.

- El método básico de enseñanza es el mecánico, autoritario e inactiva, donde el profesor dicta sus clases bajo un régimen de disciplina a unos estudiantes que son básicamente receptores.

$>\quad$ El modelo exige para educar respeto, honestidad y disciplina.

La Metodología se basa en la transmisión de la información que narra y expone el profesor.

Se explican los contenidos refiriéndose a una realidad fragmentada, estática y detenida.

Cuando se dan los temas a los estudiantes, estos no tienen por qué dar ninguna interpretación deformada de la misma.

La transmisión verbal de conocimientos es la forma habitual de enseñar en las instituciones educativas.

El uso de recursos o medios audiovisuales no cambia o altera el modelo tradicional de enseñanza, por muy modernos que estos sean.

La información transita esencialmente del profesor al alumno caracterizada por ser unidireccional por lo que no existe un verdadero proceso de comunicación.

Se trabaja en horarios prefijados, con roles de exámenes y cuidados en sus horas de descanso. 


\section{Evaluación}

Para evaluar se pide al estudiante que escuche atentamente las explicaciones, desarrolle los ejercicios, estudie memorizando y luego repase la lección y reproduzca lo más fielmente posible, lo que se exige en el examen.

$>\quad$ Está centrada en "recordar" los contenidos transmitidos en el proceso de enseñanza y de la lógica básica del discurso o del libro de texto.

> Las respuestas de los estudiantes a los exámenes dan una idea aproximada de sus aprendizajes.

$>\quad$ Se mide el aprendizaje de los diferentes estudiantes, con el mismo examen de contenidos.

> Las calificaciones por si solas provocan mecanismos de recuperación en los estudiantes, obligándolos a estudiar de nuevo los temas y contenidos no superados.

$>\quad$ Se evalúa sobre todo el resultado y a un nivel reproductivo.

$>\quad$ Las calificaciones y las notas son indicadores aceptables para medir y cuantificar los aprendizajes.

La evaluación predominantemente es escrita y se realiza mediante exámenes.

Se aplica la evaluación sumativa.

En la evaluación el profesor califica las pruebas del estudiante, ponderando las respuestas según la semejanza con el contenido expuesto.

\section{La investigación científica en la Universidad}

La educación universitaria es esencial para crear la capacidad intelectual de producir y utilizar conocimientos, y para el aprendizaje permanente que requieren las personas para actualizar sus conocimientos y habilidades. Esto es sumamente relevante ahora que vivimos en una sociedad en la que el conocimiento es el principal motor de desarrollo y crecimiento económico. Pero, para que una persona posea capacidad de producir conocimientos, y de aprendizaje permanente se requiere tener capacidades para la investigación.

Investigar es realizar actividades intelectuales y experimentales de modo sistemático con el propósito de aumentar los conocimientos sobre una determinada materia. Entonces, se considera investigación desde la indagación sistemática y organizada hasta la práctica experimental a través de la cual se genere nueva información y posterior conocimiento. José Cegarra (2009, p.41) señala: "La investigación es un proceso creador de mediante el cual la inteligencia humana busca nuevos valores. Su fin es enriquecer los distintos conocimientos del hombre, provocando acontecimientos que le hablan el por qué de las cosas, penetrando en el fondo de ellas con mentalidad exploradora de nuevos conocimientos".

De acuerdo a esto, la investigación es una función fundamental de la universidad; constituye un elemento importantísimo en el proceso educativo porque a través de ella se genera conocimiento y se propicia el aprendizaje para la generación de nuevo conocimiento; además, la investigación vincula la universidad con la sociedad. Al ser una función fundamental, sustantiva, la investigación es un deber ser. Por esta razón, las universidades deben desarrollar capacidades para la investigación en los estudiantes e incorporar la investigación como estrategia de enseñanza aprendizaje en el currículo. Guerrero (2009), define formación para la investigación: "Al conjunto de acciones orientadas a favorecer la apropiación y desarrollo de los conocimientos, habilidades y actitudes necesarios para que estudiantes y profesores puedan desempeñar con éxito actividades productivas asociadas a la investigación científica, el desarrollo tecnológico y la innovación, ya sea en el sector académico o en el productivo".

La investigación formativa en cambio, se refiere a la investigación como herramienta del proceso enseñanza aprendizaje, es decir su finalidad es difundir información existente y 
favorecer que el estudiante la incorpore como conocimiento (aprendizaje). La investigación formativa también puede denominarse la enseñanza a través de la investigación, o enseñar usando el método de investigación. La investigación formativa tiene dos características adicionales fundamentales: es una investigación dirigida y orientada por un profesor, como parte de su función docente y los agentes investigadores no son profesionales de la investigación, sino sujetos en formación.

Las universidades deben ser conscientes que la mayoría de sus egresados no harán investigación como su actividad principal, sin embargo, deben tener la capacidad de hacer investigación y realizarla cuando sea necesaria en su actividad laboral.

Por esta razón, algunas universidades están implementando programas trasversales como el Programa Institucional de Semilleros de Investigación. El semillero de investigación es un ambiente diseñado para identificar y reafirmar la vocación investigativa en general o en un campo o área específica, está integrado por un investigador de carrera que lo lidera, y un grupo de estudiantes o profesores que desean iniciarse en la investigación.

Los semilleros generalmente asumen el desarrollo de seminarios de investigación, talleres, foros, mesas redondas, paneles, charlas y sesiones de discusión, como actividades colectivas; mientras que los trabajos de documentación, lectura y análisis de documentos, generación de escritos, diseño de esquemas y prototipos, y formulación de proyectos, son ejecutados en forma individual, pero cotejados y sustentados en sesiones de trabajo presencial y grupal.

Los semilleros de investigación, formados en su mayoría por estudiantes, constituyen un nuevo modelo de enseñanza aprendizaje. Se conciben como un espacio para ejercer la libertad y la crítica académica, la creatividad y la innovación. Un semillero no sólo genera conocimiento para el mejoramiento de los sistemas, sino que transfiere y capacita a sus integrantes para el desarrollo de pensamiento. Una universidad que posea políticas adecuadas para la generación de proyectos y programas de investigación, y exista una organización de la investigación, la institución, los docentes y los estudiantes tienen alta posibilidad de beneficiarse, haciendo parte de los objetivos, formas de trabajo, equipamiento de laboratorio, formación académica y otros factores involucrados, accediendo a través de trabajos de grado, seminarios, conferencias, publicaciones, foros, etc., al desarrollo de conocimiento, productividad técnico - científica y aplicaciones que beneficien en una mayor dimensión profesional a la sociedad, como los proyectos de investigación. De esto se deduce que es beneficioso para las universidades apoyar el desarrollo de semilleros de investigación porque fortalecen la investigación formativa, experimental, descriptiva y otros tipos de investigación de acuerdo con el capital humano y por ende el producto profesional que entrega a la sociedad.

El trabajo de los estudiantes con los docentes que investigan se llamaría investigación formativa, y trabajando cerca de un profesor o de grupo de investigadores, se aprende, entre muchas cosas a:
a) Plantear problemas.
d) Diseñar metodología.
b) Formular hipótesis.
e) Trabajar en equipo.
c) Saber recopilar información y
f) Discutir resultados. sintetizarla.

Y, además de la formación en investigación o de la investigación formativa, ayuda a:
a) Fortalecer los espacios académicos.
b) Familiarizar al estudiante y al docente con métodos y técnicas investigativas.
c) Servir de ensayo y experimentación en procesos investigativos y de aprendizaje. 


\section{RESULTADOS}

A. ANÁLISIS DEL ESTILO TRADICIONAL

\section{Tabla 1: Niveles de aplicación del estilo didáctico tradicional en los docentes}

\begin{tabular}{|c|c|c|}
\hline $\begin{array}{l}\text { Niveles de aplicación del estilo } \\
\text { didáctico tradicional }\end{array}$ & Frecuencia & Porcentaje \\
\hline BAJA APLICACIÓN & 7 & 23.3 \\
REGULAR APLICACIÓN & 15 & 50.0 \\
ALTA APLICACIÓN & 8 & 26.7 \\
Total & 30 & 100.0 \\
\hline
\end{tabular}

Fuente: Cuestionario a estudiantes

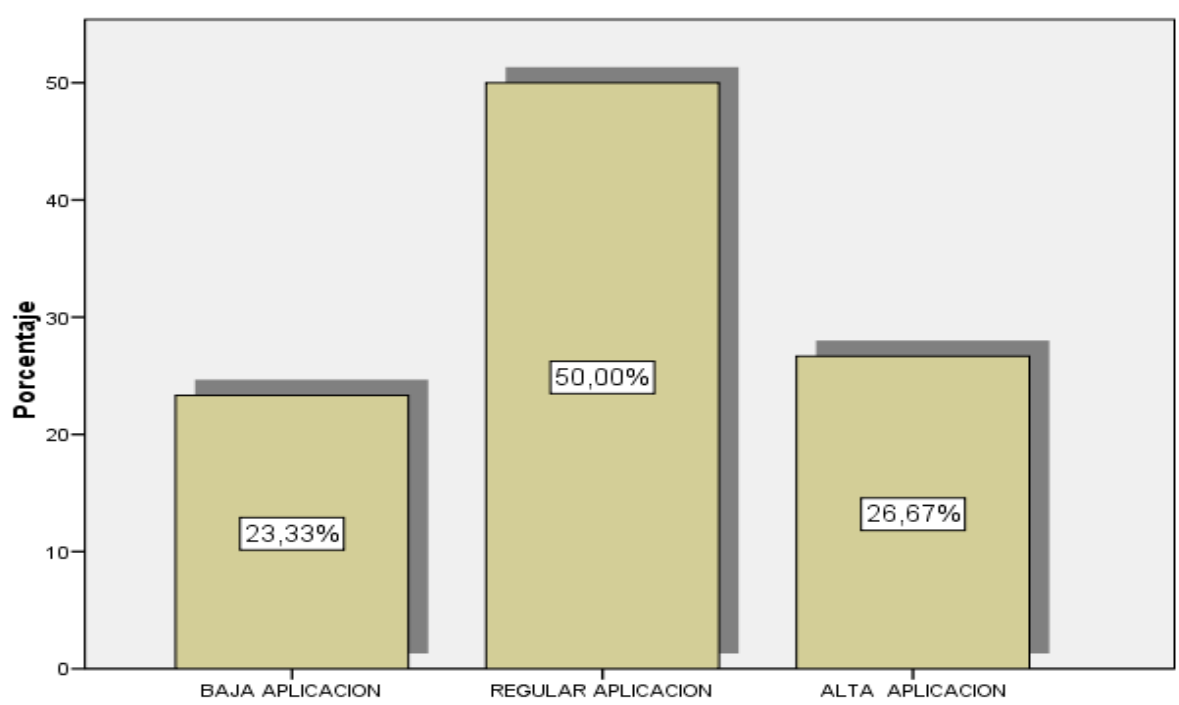

Figura 1 : Niveles de aplicación del estilo didáctico tradicional

Análisis

La tabla 1 y figura 1, permite comprobar que el $76.7 \%$ de docentes de la Escuela Profesional de Ingeniería Comercial practican el estilo didáctico tradicional con fuerte énfasis en el contenido.

Con el valor de chi-cuadrado 0.01 (siendo el valor de $p=0,05$ ), se verifica la hipótesis de que los docentes de la Escuela Profesional de Ingeniería Comercial aplican el estilo didáctico tradicional basado en contenidos, con un nivel de confianza del $95 \%$. 
Tabla 2: Niveles de desarrollo de capacidad de investigación

\begin{tabular}{|c|c|c|}
\hline NIVELES DE CAPACIDAD & Frecuencia & Porcentaje \\
\hline $\begin{array}{l}\text { BAJA CAPACIDAD DE } \\
\text { INVESTIGACIÓN }\end{array}$ & 7 & 23.3 \\
$\begin{array}{l}\text { REGULAR CAPACIDAD } \\
\text { DE INVESTIGACIÓN } \\
\text { ALTA CAPACIDAD DE } \\
\text { INVESTIGACIÓN }\end{array}$ & 19 & 63.3 \\
Total & 4 & 13.3 \\
\hline
\end{tabular}

Fuente: Cuestionario a estudiantes

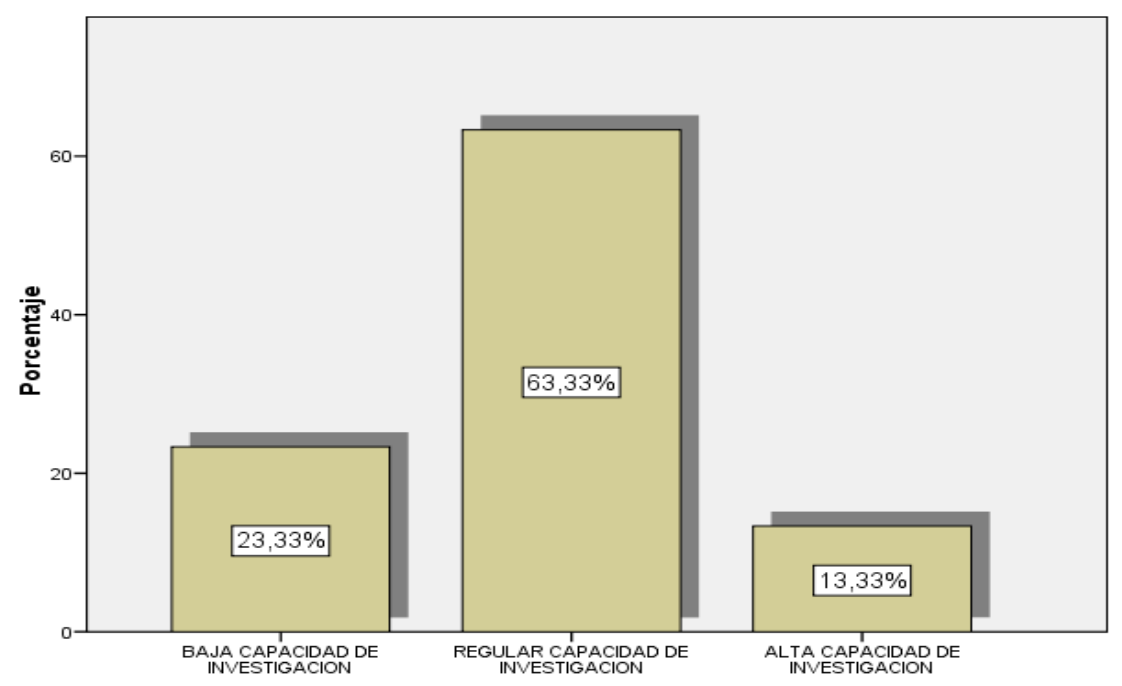

Figura 2 : Niveles de desarrollo de capacidad de investigación

Análisis

La tabla 2 y figura 2, permite comprobar que el $96.67 \%$ de docentes de la Escuela Profesional de Ingeniería Comercial no ponen en práctica en sus clases la elaboración de notas científicas generando un bajo nivel de desarrollo de la capacidad de investigación científica de los estudiantes.

Con el valor de chi-cuadrado 0.045 (siendo el valor de $p=0,05$ ), se verifica la hipótesis de que los docentes de la Escuela Profesional de Ingeniería Comercial no desarrollan trabajos de investigación científicas, con un nivel de confianza del 95\%. 
Tabla 3: Implicancias de la didáctica tradicional en la capacidad de investigación de los estudiantes

\begin{tabular}{|l|l|l|l|l|}
\hline Variables & $\begin{array}{l}\text { Chi-cuadrado } \\
\text { Calculado }\end{array}$ & $\begin{array}{l}\text { P-valor } \\
\text { teórico }\end{array}$ & Resultado & Implicancias \\
\hline $\begin{array}{l}\text { Estilo didáctico } \\
\text { tradicional }\end{array}$ & $\chi^{2}=0.001$ & $\mathrm{P}=0.05$ & $\begin{array}{l}\text { Predominio del } \\
\text { estilo didáctico } \\
\text { tradicional }\end{array}$ & $\begin{array}{l}\text { El docente } \\
\text { enseña en } \\
\text { base a los } \\
\text { contenidos }\end{array}$ \\
\hline $\begin{array}{l}\text { Capacidad de } \\
\text { investigación }\end{array}$ & $\chi^{2}=0.045$ & $\mathrm{P}=0.05$ & $\begin{array}{l}\text { Predominio de } \\
\text { clases teóricas }\end{array}$ & $\begin{array}{l}\text { El estudiante } \\
\text { no desarrolla } \\
\text { su capacidad } \\
\text { de } \\
\text { investigación } \\
\text { científica }\end{array}$ \\
\hline
\end{tabular}

En la tabla 3 se resume las implicancias del estilo didáctico tradicional en la capacidad de investigación de los estudiantes en la Escuela Profesional de Ingeniería Comercial de la Facultad de Ciencias Empresariales de la Universidad Privada de Tacna.

\section{CONCLUSIONES}

a) Los profesores universitarios de la Facultad de Ciencias Empresariales practican el estilo didáctico tradicional que se basa en la implementación y desarrollo de los contenidos establecidos en los sílabos por curso.

b) Los profesores universitarios de la Facultad de Ciencias Empresariales desarrollan actividades de aprendizaje solamente para asegurar el logro de competencias académicas en el tiempo establecido en el sílabo.

c) Los profesores universitarios de la Facultad de Ciencias Empresariales no desarrollan actividades de investigación científica en el aula generando un limitado desarrollo de la capacidad de investigación en los estudiantes.

d) Las implicancias del predominio del estilo didáctico tradicional se manifiesta en una enseñanza basada en el contenido y en una escasa producción científica por parte de los estudiantes.

\section{REFERENCIAS BIBLIOGRAFÍCAS}

- Cegarra Sánchez, José. (2004). Metodología de la investigación científica y tecnológica. Edic. Días Santos.

- $\quad$ ABC del educador.(2003). Modelos Pedagógicos y Didácticos. Bogotá: Ed. SEM.

- Caviedes Hoyos, Raymundo.(2007). Importancia del Ensayo para la Maestría. Documento, aportado como guía, Marcos Turbay. Uninorte.

- Cruz García, Rolando, (2008) La didáctica, hacia dónde va el arte de enseñar. Disponible en: http://www.buenastareas.com/ensayos/La-Didactica-Tradicional/12014.html

- Documento: Estándares Básicos de Competencias. (2007). MEN. Bogotá.

- Flores Ochoa, Rafael. (2005). Pedagogía del Conocimiento. Bogotá. Segunda Edición. Ed. Mc Graw Hill.

- García, Pérez, Francisco. (2008) Los modelos didácticos como instrumento de análisis de intervención en la realidad educativa. Disponible en: http://www.ub.es/geocrit/b3w-207.htm

- Marqués, Graells, Pere (2010), La Tecnología Educativa; conceptualización, líneas de investigación. Disponible en: http://peremarques.pangea.org/tec.htm

Recibido : 26/4/2016

Aceptado para publicación $15 / 5 / 2016$ 